\title{
Change of Ascorbic Acid Level after Grafting of Tomato Seedlings
}

Akira Wadano $^{a}$,*, Mitsuharu Azeta ${ }^{a}$, Shin-ichi Itotani ${ }^{\mathrm{a}}$, Ai Kanda ${ }^{\mathrm{a}}$, Toshio Iwaki ${ }^{\mathrm{a}}$, Tomoaki Taira $^{\mathrm{b}}$, Yasushi Fujii ${ }^{\mathrm{b}}$, Yoshifumi Nishiura ${ }^{\mathrm{c}}$, Haruhiko Murase ${ }^{\mathrm{c}}$ and Nobuo Honami ${ }^{\mathrm{c}}$

a Department of Applied Biochemistry

b Department of Plant Science

c Department of Regional Environmental Science, College of Agriculture, Osaka Prefecture University, Sakai 599-8531, Osaka, Japan. Fax: 81-722-54-9921. E-mail: wadano@center.osakafu-u.ac.jp

* Author for correspondence and reprint requests

Z. Naturforsch. 54c, 830-833 (1999); receivced December 20, 1998/February 3, 1999

Ascorbate Peroxidase, Ascorbic Acid, Glutathione, Grafting, Water Stress

Grafting is an easy way to produce a new seedling, which can tolerate against various stresses. During the acclimation after grafting, however, the seedlings still suffer a severe water stress. It is well known that water stress produces active oxygen to oxidize ascorbic acid. The concentration of ascorbic acid in the leaves was analyzed by HPLC equipped with an electrochemical detector. The column used was SP-120-5-ODS-BP (DAISO, JAPAN) and elution was performed with $0.1 \mathrm{~m}$ phosphate buffer, $\mathrm{pH}$ 3.0. After grafting the seedlings were acclimated under a 6-hr light/dark regimen. The content of ascorbic acid increased gradually during 2 days compared with control. The ascorbate peroxidase showed about constant activity, so the increase of ascorbic acid may be due to its requirement to cure the grafting.

\section{Introduction}

Grafting is an easy way to produce new seedlings, which can tolerate various stresses. A novel grafting robot (plug-in-method robot) performed better joining than those obtained by the "cleft" or the "cutting" method (Honami et al., 1992). During acclimation after grafting, however, the seedlings still suffer a severe water stress. It is well known that water stress produces active oxygen to oxidize ascorbic acid and glutathione. Ascorbic acid also is involved in cell wall metabolism and cell expansion (Smirnoff, 1996). Therefore, plants need some ascorbic acid during the acclimation after grafting. On the other hand, the determination of ascorbic acid and dehydroascorbic acid have been challenged, and Lykkesfeldt et al. (1995) reported the analysis of the compounds by high-performance liquid chromatography (HPLC) with coulometric detection. They obtained clear data of the amount of ascorbic acid and dehydroascorbic acid in plasma, but the retention time of ascorbic acid was short. The analysis is not easy if the samples contain any ionic contamination, which elutes at the solvent front. We therefore utilized a new column to analyze ascorbic acid and to check its concentration during the acclimation of grafted tomato seedlings in this study.

\section{Materials and Methods}

\section{Tomato plant}

The scion and stocks were "Momotarou" and "LS89", respectively. The seedlings for grafting were grown by planting single tomato seeds in a "System soil 102" (Iwatani Co. Ltd., Japan). The seeds in a tray were left to germinate and grow at $20-25{ }^{\circ} \mathrm{C}$ in a greenhouse for about $30-40$ days, and were irrigated by a bottom method. The growth stage of scions was $2-3$ leaves old and $1.5-2.8 \mathrm{~mm}$ in stem diameter, while that for the stocks was 3-4 leaves old and $1.8-3.0 \mathrm{~mm}$ in stem diameter.

\section{Plug-in method}

The new grafting method produces a taper tip for scion and a conical hole for stock. This method provides high transport of water and nutrients between scion and stock, since a large area of joining surface gives a high degree of contact. 


\section{Chromatographic analysis}

Concentration of ascorbic acid of the leaves was analyzed by HPLC equipped with electrochemical detector. The HPLC system consisted of GP-A50 gradient programmer, 851-AS intelligent sampler, BIP-1 HPLC pump (JASCO, Japan) and chromatogram processor model 7000S (SIC, Japan). The column used was SP-120-5-ODS-BP (DAISO, JAPAN) and elution was performed with $0.1 \mathrm{~m}$ phosphate buffer, pH 3.0 at $1 \mathrm{ml} / \mathrm{min}$. For peak detection, the chromatographic system was connected to an AMPEROMETER $\Sigma 875$ (IRICA, Japan) operated at $0.1 \mathrm{~V}$.

\section{Extraction of ascorbic acid}

After grafting the seedlings was acclimated under a 6-hr light/dark regimen. For the complete acclimation, ca. $120 \mathrm{hr}$ was required. Relative photosynthetic activity recovered gradually after $90 \mathrm{hr}$, although height and water content of the grafted seedling did not increase. During acclimation, leaves were detached at fixed days to analyze for ascorbic acid. The detached leaves were frozen with liquid nitrogen, then homogenized with pestle and stainless steel bowl. The powder was mixed with $1.0 \mathrm{~m}$ phosphate buffer, $\mathrm{pH} 0.7$, then centrifuged to remove cell debris for $5 \mathrm{~min}$ at $24,000 \times g$. The supernatant was used as the sample for analysis of reduced ascorbic acid. Total ascorbic acid was measured after reduction of the dehydroasc- orbic acid (Lykkesfeldt et al., 1995). The reduction was carried out adding $200 \mu \mathrm{l}$ of the sample to $65 \mu \mathrm{l}$ of $50 \mathrm{~mm}$ DTT and $35 \mu \mathrm{l}$ of $6 \mathrm{~N} \mathrm{NaOH}$, and kept standing at $37^{\circ} \mathrm{C}$ for $5 \mathrm{~min}$ in the dark. Then, $100 \mu \mathrm{l}$ of $2 \mathrm{M} \mathrm{H}_{2} \mathrm{SO}_{4}$ were added to the reduced sample and the mixture was used for the analysis of total ascorbic acid.

\section{Determination of Chl content and quantitation of protein}

Chl was estimated spectrophotometrically by the method of Mackinney (1941) after extraction with $80 \%$ acetone. Protein was quantitated by the method of Bradford (1976) with crystalline bovine serum albumin as standard.

\section{Assay of ascorbate peroxidase}

Hundred $g$ of tomato leaves were homogenized with pestle and stainless steel bowl. The powder was mixed with a grinding buffer consisting of $1 \mathrm{~mm}$ ascorbic acid, $1 \mathrm{~mm}$ EDTA, and $50 \mathrm{~mm}$ potassium phosphate buffer $(\mathrm{pH} 7.0)$, then centrifuged for $10 \mathrm{~min}$ with $12,000 \times g$ at $4{ }^{\circ} \mathrm{C}$. The supernatant was desalted with Sephadex G-10, and the eluate used as the enzyme solution. The reaction mixture consisted of $50 \mathrm{~mm}$ potassium phosphate buffer ( $\mathrm{pH} 7.0$ ), $0.5 \mathrm{~mm}$ ascorbic acid and $0.1 \mathrm{~mm} \mathrm{H}_{2} \mathrm{O}_{2}$ in a total volume of $1 \mathrm{ml}$. The reaction was started with the addition of the enzyme solution. Enzyme activity was assayed by measur-
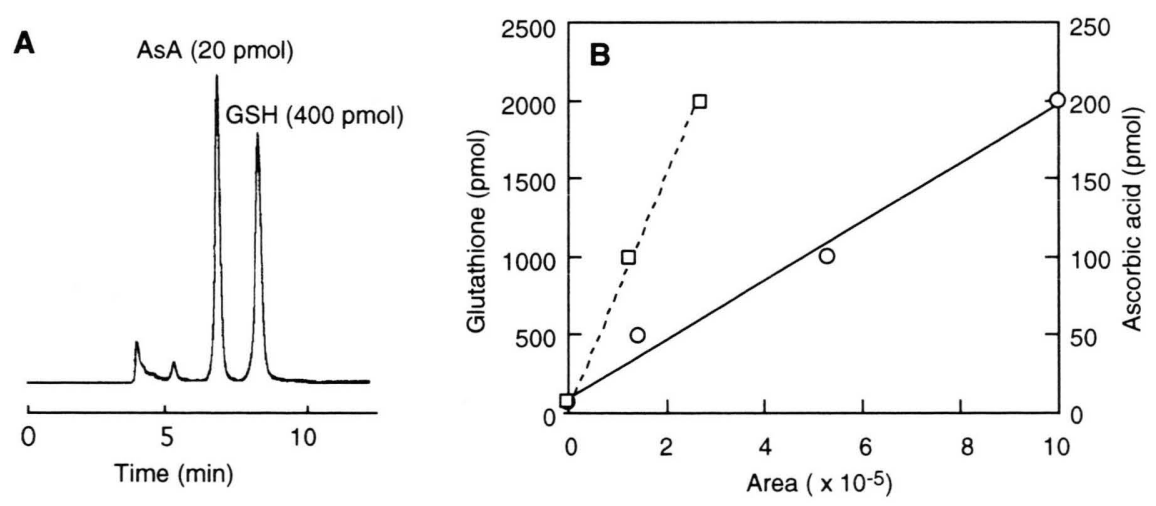

Fig. 1. Analyses of ascorbic acid (AsA) and reduced glutathione (GSH) by ECD-HPLC with an ODS-column, DAISOPACK SP-120. The HPLC system consisted of GP-A50 gradient programmer, 851-AS intelligent sampler, BIP-1 HPLC pump (JASCO, Japan) and chromatogram processor Model 7000S (SIC, Japan). The elution was performed with $0.1 \mathrm{~m}$ phosphate buffer, pH 3.0 at $1 \mathrm{ml} / \mathrm{min}$. For peak detection, the chromatographic system was connected to an AMPEROMETER $\Sigma 875$ (IRICA, Japan) operated at $1.0 \mathrm{~V}$. A; elution profile, B; the sensitivity of the analysis. Circles; reduced ascorbic acid, squares; reduced glutathione. Area, arbitrary unit by chromatogram processor. 
ing the decrease of absorbance of ascorbic acid at $290 \mathrm{~nm}$ with a Hitachi U-3210 spectrophotometer. The millimolar extinction coefficient of ascorbic acid was $2.8 \mathrm{~mm}^{-1} \mathrm{~cm}^{-1}$ at $290 \mathrm{~nm}$.

\section{Results and Discussion}

\section{Analysis of ascorbic acid}

The method can detect 10 to 200 pmol of ascorbic acid. The retention time of ascorbic acid and glutathione on HPLC under the conditions mentioned above are about 5 and 6.5 min, respectively. The sensitivity for ascorbic acid is one order higher than that of glutathione. The sample contained ions, which elute on the solvent front, but the peak of ascorbic acid is still clear. When the applied voltage of the electrochemical detector was reduced to $0.1 \mathrm{~V}$, the system was highly specific against ascorbic acid. The method can be applied to analyze the content of ascorbic acid in the plant (Fig. 1).

\section{Estimation of ascorbic acid contents}

Ascorbic acid increased sharply up to $8 \mathrm{hrs} \mathrm{com-}$ pared with the control (Fig. 2). Thirty hr after grafting the content of the compound decreased to the level of the control. Grafting caused the water stress, but it was not so detectable up to $7 \mathrm{hr}$. Therefore, the increase of the ascorbic acid

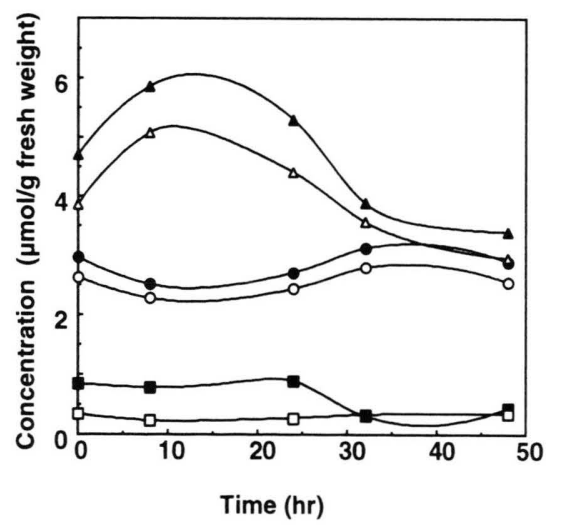

Fig. 2. Change of ascorbic acid and dehydroascorbic acid contents after grafting of tomato seedlings. Total ascorbic acid was measured after reduction of the dehydroascorbic acid as described in text. The values are the mean of duplicate experiments. Open symbols are for control and closed symbols are for the grafted seedlings. Triangle; total ascorbic acid, circle; reduced ascorbic acid, square; dehydroascorbic acid. content was not due to decrease of the water content. The ascorbic acid content of the scion seemingly was restored to normal around $30 \mathrm{hr}$ after grafting, although $\mathrm{CO}_{2}$ fixation rates turned back to the normal gradually from 72 to $96 \mathrm{hr}$ (Nishiura et al., 1996). It is not clear what causes the increase of the ascorbic acid content.

\section{Ascorbate peroxidase}

Ascorbate peroxidase activity was almost constant during acclimation (Fig. 3). The activity of the soluble fraction was about the same as that of the spinach. But the activity bound to thylakoid membranes could not be detected by the differential inactivation method of Amako et al. (1994). The constant activity of the ascorbate peroxidase implies that the activity had little relationship to the increase of ascorbic acid during acclimation after grafting. Since the $\mathrm{Km}$ values for ascorbic acid of the peroxidase are in the $\mu \mathrm{M}$-range (De Gara et al., 1997), the change of ascorbic acid concentration in $\mu \mathrm{m} / \mathrm{g}$ fresh wt may not affect the peroxidase. Smirnoff (1996) showed that the ascorbic acid is required to recover from the wounding of plant. Grafting should make the same signal as wounding, so signal to increase of ascorbic acid content may originate from cell wall metabolism and cell expansion.

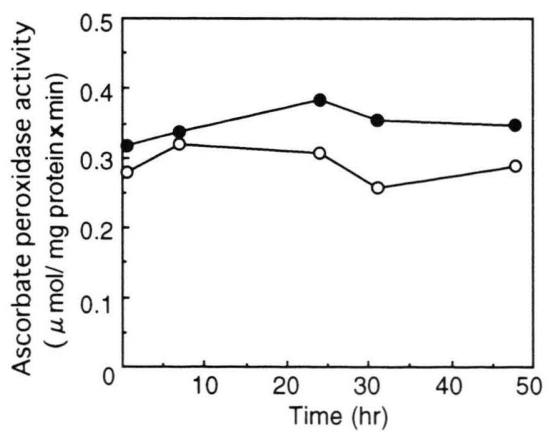

Fig. 3. Change of ascorbate peroxidase activity after grafting of tomato seedlings. Enzyme activity was assayed by measuring the decrease of light absorbance of ascorbic acid at $290 \mathrm{~nm}$ with a Hitachi U-3210 spectrophotometer. Open circles: control, closed circles: grafted seedlings. 
Amako K., Chen G.-X. and Asada K. (1994), Separate assays specific for ascorbate peroxidase and guaiacol peroxidase and for the chloroplastic and cytosolic isozymes of ascorbate peroxidase in plants. Plant Cell Physiol. 35, 497-504.

Bradford M. M. (1976), A rapid and sensitive method for the quantitation of microgram quantities of protein utilizing the principle of protein dye binding. Anal. Biochem. 72, 248-254.

De Gara L., de Pinto M. C. and Arrigoni O. (1997), Ascorbate synthesis and ascorbate peroxidase activity during the early stage of wheat germination. Physiol. Plant. 100, 894-900.

Honami N., Taira T., Murase H., Nishiura Y. and Yasukuri Y. (1992), Robotization in the production of grafted seedlings. Acta Horticult. 319, 579-584.
Lykkesfeldt J., Loft S. and Poulsen H. E. (1995), Determination of ascorbic acid and dehydroascorbic acid in plasma by high-performance liquid chromatography with coulometric detection - Are they reliable biomarkers of oxidative stress? Anal. Biochem. 229 $329-335$.

Mackinney G. (1941), Absorption of light by chlorophyll solutions. J. Biol. Chem. 140, 315-322.

Nishiura Y., Murase H., Honami N., Taira T. and Wadano A. (1996), Carbon dioxide fixation rate characteristics of a grafted seedling in an acclimation process. Acta Horticult. 440, 251-255.

Smirnoff N. (1996), The function and metabolism of ascorbic acid in plants. Ann. Bot. 78, 661-669. 